Faculté des sciences sociales École de psychologie

\title{
PRATIQUE EXCESSIVE DES JEUX VIDÉO CHEZ LES PERSONNES AYANT UN TROUBLE DU SPECTRE DE L'AUTISME
}

\author{
Kathia COUTURE ${ }^{1 *}$ \\ 'École de psychologie, Université Laval, Québec, QC, Canada \\ *Kathia.couture.1@ulaval.ca
}

\section{Pour citer l'article}

Couture, K. (2020). Pratique excessive des jeux vidéo chez les personnes ayant un trouble du spectre de l'autisme. Psycause: Revue scientifique étudiante de l'École de psychologie de I'Université Laval, 10(1), 18-27.

\section{Droits d'auteur}

(C) 2020 Couture. Cet article est distribué en libre accès selon les termes d'une licence Creative Commons Attribution 4.0 International (de type CC-BY 4.0) qui permet l'utilisation du contenu des articles publiés de façon libre, tant que chaque auteur ou autrice du document original à la publication de l'article soit cité(e) et référencé(e) de façon appropriée. 


\title{
PRATIQUE EXCESSIVE DES JEUX VIDÉO CHEZ LES PERSONNES AYANT UN TROUBLE DU SPECTRE DE L'AUTISME
}

\author{
Kathia COUTURE ${ }^{1 *}$ \\ 'École de psychologie, Université Laval, Québec, QC, Canada \\ *Kathia.couture.1@ulaval.ca
}

\begin{abstract}
Résumé
Les symptômes diagnostic du trouble du spectre de l'autisme (TSA), tels que décrits dans la cinquième édition du Manuel Diagnostique et Statistiques des Troubles Mentaux (DSM), incluent des déficits dans l'interaction sociale et dans la réciprocité sociale et émotionnelle ainsi qu'un caractère répétitif et stéréotypé des comportements et des intérêts. La technologie numérique telle que les jeux vidéo est utilisée comme traitement en psychothérapie pour pallier ces déficits. Chez des individus neurotypiques, c'est-à-dire des individus n'ayant pas un TSA, l'utilisation de jeux vidéo entraînerait plusieurs effets, dont des changements neuropsychologiques et neurologiques. Dans certains cas, une utilisation excessive ou pathologique est observée chez des individus neurotypiques. Ainsi, il est important de se questionner si des impacts similaires peuvent être retrouvés chez les individus avec un TSA. Toutefois, certains chercheurs dans ce domaine suggèrent que l'utilisation de ces technologies numériques, plus spécifiquement la réalité virtuelle, entraînerait une amélioration de leurs compétences sociales, du décodage non verbal, du contrôle des stimuli ainsi que de la compréhension chez les individus avec un TSA. De même, la réalité virtuelle améliorerait, toujours chez la population avec un TSA, des mesures sociales et cognitives de la théorie de l'esprit et de la reconnaissance des émotions. Cependant, d'autres mentionnent des risques d'addiction et d'isolement social qui seraient peut-être même plus grands que ceux retrouvés chez les individus neurotypiques. En bref, cette revue de la littérature étudie le risque de pratique excessive des jeux vidéo chez les individus avec un TSA.
\end{abstract}

\section{Mots-clés: Trouble du spectre de l'autisme, Jeux vidéo, Dépendance aux jeux vidéo, Utilisation excessive des jeux vidéo, Neurotypique}

\section{Abstract}

The diagnostics symptoms of autism spectrum disorder (ASD), as described in the fifth edition of the Diagnostic and Statistical Manual of Mental Disorders (DSM), includes social and emotional deficits, social interaction deficits as well as adhering to repetitive and stereotypical behaviors and interests. Innovative technologies such as video games are used in psychotherapy to overcome those deficits. Video games when used by neurotypicals tend to lead to neuropsychological and neurological changes and sometimes excessive use. Therefore, it is possible that these changes also occur in ASD individuals. Some research in this field of study suggest that the use of innovative technology and more specifically virtual reality tend to improve social skills, non-verbal decoding, and stimulus control and comprehension for the ASD population. Virtual reality could also improve social and cognitive measures related to theory of mind and emotion recognition. Conversely, other studies underline the potentially higher risks of addiction and isolation in ASD versus neurotypical individuals. In short, this literature review studies the risk of excessive playing of video games for individuals with ASD.

\section{Keywords : Autism spectrum disorder, Video games, Video game addiction, Excessive video gaming, Neurotypical}

Ce texte se veut être une revue de la littérature qui brosse un portrait global des études faites dans le domaine de la pratique excessive des jeux vidéo (JV) auprès des individus ayant un trouble du spectre de l'autisme (TSA). De cette manière, d'une part, il y a une remise en contexte de la symptomatologie du
TSA, de son diagnostic et de sa prévalence dans la population ainsi qu'une présentation de l'utilisation des JV en contexte thérapeutique et en contexte de divertissement. D'autre part, une association est présentée entre les caractéristiques des individus ayant une dépendance aux JV en ligne et les caracté- 
ristiques des individus avec un TSA. Finalement, i y est présenté des études sur des conséquences neuropsychologiques et neurologiques des JV sur des individus neurotypiques, c'est-à-dire des individus n'ayant pas un TSA, et des individus avec un TSA, et ce, en prenant en compte les particularités cérébrales de ces derniers.

\section{La symptomatologie et le diagnostic du TSA}

La première description du TSA revient à Kanner en 1943. Kanner parle d'un syndrome de l'autisme infantile qu'il considère d'abord comme un syndrome psychopathologique, puis qu'il redéfinit comme un syndrome clinique. Depuis, la définition ainsi que la conceptualisation de l'autisme ont énormément évolué (Bolduc \& Poirier, 2017). La cinquième édition du Manuel Diagnostique et Statistique des Troubles Mentaux (DSM) publiée en 2013 réorganise entièrement le procédé diagnostic. Selon le DSM-5, le TSA renvoie à une notion de spectre ayant deux sphères de symptômes, soit l'altération sociocommunicationnelle et le caractère répétitif et stéréotypé des comportements et des intérêts, variant de légers à sévères (APA, 2013). Les symptômes doivent être présents dès la petite enfance et limiter, voire altérer, le fonctionnement quotidien de l'individu dans divers contextes de vie comme la sphère scolaire, familiale et/ou professionnelle (APA, 2013). Il est toutefois possible que l'altération du fonctionnement de la personne n'apparaisse que plus tard dans le développement de l'individu, c'est-à-dire seulement lorsque la demande sociale excède les capacités de l'individu, notamment lors de la rentrée scolaire ou bien à l'adolescence (Bolduc \& Poirier, 2017). Une fois le diagnostic établi, il est important d'y apporter quelques précisions dans le but de mettre en place des interventions appropriées. D'emblée, il est nécessaire d'estimer le degré de sévérité de l'atteinte, soit si elle est «légère », «modérée » ou "sévère" en fonction du besoin de soutien de I'individu dans les deux sphères de symptômes. Enfin, le TSA est souvent en comorbidité avec d'autres troubles, tels qu'un retard intellectuel, des difficultés langagières, une condition médicale ou génétique, de la catatonie ou un autre trouble développemental (APA, 2013).

\section{La prévalence et les programmes thérapeutiques du TSA}

Au cours des dernières années, la prévalence du TSA ne fait qu'augmenter. Ce phénomène s'explique, en partie, par l'élargissement des critères diagnostiques et par une meilleure connaissance du trouble. Les études épidémiologiques actuelles suggèrent que le TSA touche environ $1 \%$ à $2 \%$ de la population parmi tous les groupes culturels et socio-économiques. Également, il toucherait cinq fois plus les garçons que les filles (APA, 2013; Bolduc \& Poirier, 2017; Zablotsky, Black, Maener, Schieve, \& Blumberg, 2015). Les données scientifiques récentes soutiennent l'hypothèse selon laquelle le TSA repose sur une base biologique dont l'origine est principalement d'ordre génétique. Cependant, les données actuelles ne permettent pas I'identification de marqueur biologique qui permettrait le dépistage du TSA. Ainsi, son diagnostic s'appuie essentiellement sur l'histoire développementale et l'observation des comportements (Bolduc \& Poirier, 2017).

Il existe des programmes thérapeutiques pour les personnes ayant un TSA; certains sont basés sur des données probantes dont l'efficacité est prouvée par des études, tandis que d'autres possèdent une efficacité encore incertaine (Eckert, 2019). En ce qui concerne les enfants d'âge scolaire et les adolescents, les programmes thérapeutiques en matière d'accompagnement et de soutien se divisent en deux catégories, soit l'encouragement pédagogique dans le contexte scolaire et les mesures thérapeutiques complémentaires (Eckert, 2019). La technologie numérique dans la psychothérapie est aussi utilisée comme modalité de traitement. Toutefois, l'efficacité de ce secteur d'activité est incertaine puisqu'il est en émergence (Grossard \& Grynszpan, 2015). Ainsi, si l'utilisation de technologies numériques et, par le fait même, des $J V$ est un domaine en émergence, qu'en est-il de I'utilisation de JV comme divertissement chez la population avec un TSA ? Autrement dit, avant d'utiliser le JV comme modalité de traitement, il est important de comprendre les effets neuropsychologiques et neurologiques des JV dans un contexte non thérapeutique chez les individus avec un TSA. D'emblée, chez les utilisateurs neurotypiques, un risque est présent quant à leur utilisation excessive. Un questionnement subsiste donc à savoir s'il existe le même risque de pratique excessive chez les individus avec un TSA. 


\section{Les jeux vidéos: du divertissement aux applicatins médicales}

À la suite d'une enquête auprès de la population française, Ludespace, Rufat, Minassian et Coavoux (2014) ont analysé l'utilisation des JV. Cette enquête est basée sur un échantillon représentatif de personnes ayant 18 ans et plus ainsi que sur un sous-échantillon représentatif de personnes ayant entre 11 et 17 ans. Les résultats montrent une forte proportion de joueurs dans la population française dont près des deux tiers pratiquent un ou plusieurs JV. Cette proportion s'élève à $97 \%$ chez les enfants et les adolescents (1117 ans) et elle excède les $90 \%$ chez les filles (Rufat, Minassian, \& Coavoux, 2014). II est possible de croire que des statistiques similaires soient retrouvées auprès de la population québécoise. Les JV sont donc un média très populaire qui touche un public large et hétérogène (Brun, 2017). De même, d'après une large étude paneuropéenne effectuée en 2010 ayant un sous-échantillon des jeunes de 13-16 ans, $80 \%$ d'entre eux utilisent I'Internet pour jouer à des jeux en ligne (Sallafranque St-Louis, 2015).

En ce sens, l'intérêt porté aux JV par la recherche en psychiatrie est plutôt focalisé. En effet, les deux thèmes principalement étudiés sont les risques addictifs et la propension à la violence des consommateurs. La popularité des JV est alors plutôt étonnante si de tels risques et une telle représentation sociale négative y sont associés. Malgré cela, il est admis que le jeu permet le développement de capacités bénéfiques à l'individu qui I'utilise, que ce soit au niveau psychique (p. ex., bien-être, hédonie), cognitif (p. ex., la mémoire ou les fonctions exécutives) ou physique (Brun, 2017).

\section{La dépendance aux jeux vidéos en ligne et les caractéristiques des individus avec un TSA}

La dépendance aux JV en ligne sur Internet est reconnue comme un trouble par l'Organisation mondiale de la Santé; il est retrouvé dans la $11^{\mathrm{e}}$ édition de la Classification statistique internationale des maladies (Organisation mondiale de la Santé, 2018). Toutefois, ce trouble n'est pas officiellement reconnu parl'American Psychiatric Association (APA). Certes, plusieurs études (Batthyány \& Pritz, 2009; Grüsser \& Thalemann, 2006, cités dans Kuss \& Griffiths, 2012) montrent qu'aux niveaux neurologique et biochimique, la dépendance aux JV sur Internet est similaire à plusieurs autres dépendances liées à une substance, ce qui soutient I'hypothèse qu'elle pourrait se classer dans la même catégorie que la dépendance au jeu d'argent, en d'autres termes, dans une toxicomanie sans substance.

II est à noter que la pathologie est concentrée sur les $\mathrm{JV}$ en ligne tandis que cette revue de la littérature se concentre sur les JV en général, c'est-à-dire qu'ils peuvent être en ligne ou non. De plus, il sera davantage question de pratique excessive des JV plutôt que de dépendance aux JV. La distinction est importante, puisque la pratique excessive des JV est considérée comme une étape menant vers la dépendance (Kuss \& Griffiths, 2012). Néanmoins, aucune étude ne semble établir de corrélation entre les traits de personnalité associés à la dépendance aux JV en ligne et à ceux retrouvés chez les joueurs ayant une pratique excessive des JV en général.

La revue systématique des recherches de Kuss et Griffiths (2012) montre que plusieurs traits de personnalité sont significativement associés à la dépendance aux JV en ligne. Ceux-ci peuvent se regrouper en trois catégories, soient l'introversion, le névrosisme et l'impulsivité. Ces catégories englobent alors des traits tels que la solitude et l'introversion, l'inhibition sociale, une faible intelligence émotionnelle ou encore " une faible efficacité dans la vie réelle par opposition à une efficacité élevée dans le monde virtuel» (Kuss \& Griffiths, 2012). II semble que les caractéristiques des individus plus à risque de développer une dépendance aux JV en ligne sont, d'une certaine manière, similaires aux caractéristiques des individus avec un TSA. Les difficultés sociocommunicationnelles retrouvées chez les individus avec un TSA peuvent être caractérisées, entre autres, par une approche sociale anormale, des déficits dans la conversation ainsi que des difficultés à partager leurs émotions et comprendre celles des autres, allant jusqu'à I'incapacité de répondre aux interactions sociales (APA, 2013). La dépendance aux JV en ligne n'est cependant pas exclusivement influencée par les traits de personnalité. En effet, la dépendance aux $\mathrm{JV}$ en ligne est également associée aux motivations intrinsèques du joueur. Celles-ci sont particulièrement étudiées auprès des adolescents et des adultes. En ce sens, il semble que la dépendance aux JV en ligne soit 
liée, de façon générale, à des difficultés d'adaptation à la vie réelle ainsi qu'à une faible socialisation (Kuss \& Griffiths, 2012).

Toutefois, dans ce contexte, il est possible que les caractéristiques corrélées avec la dépendance aux JV en ligne ne soient pas les mêmes que pour les autres types de JV (p. ex. les JV de tir à la première personne, de rôle ou encore d'horreur). De plus, il est possible que pour chaque type de JV, il y ait d'autres traits de personnalité ou de motivations qui y sont associés.

\section{Conséquences des jeux vidéos chez des sujets neurotypiques}

\section{Les conséquences neuropsychologiques chez des sujets neurotypiques}

De nombreuses recherches ont démontré que les JV ont un impact cognitif important sur ses utilisateurs. En ce sens, plusieurs études présentent une amélioration de certaines fonctions cognitives à la suite de leur utilisation. La revue de la littérature de Brun (2017), qui étudie l'utilisation des JV dans les soins de réhabilitation des sujets souffrant de schizophrénie, possède une section concernant les impacts neurologiques et neuropsychologiques des JV auprès des sujets sains.

Il est important de considérer que les fonctions cognitives améliorées varient en fonction du JV utilisé. Ainsi, les JV de tir à la première personne (first person shooter), souvent critiqués pour leur contenu violent, semblent améliorer les fonctions cognitives associées à la vision, telles que «la mémoire visuelle de travail, les perceptions de détails et de nuances de gris, la représentation et l'orientation dans l'espace, mais aussi les capacités d'attention avec un bénéfice sur l'apprentissage» (Brun, 2017, p. 18). De même, les JV sont utilisés dans un objectif d'optimisation des capacités chez des sujets neurotypiques. L'armée de l'air des États-Unis d'Amérique utilise différents JV (Glass, Maddox, \& Love, 2013) dont des jeux de stratégies en temps réel afin d'améliorer les capacités de concentration et de transfert entre les différentes informations perçues, ce qui optimiserait la prise de décision rapide (fast-thinking; Brun, 2017).

Or, certains effets négatifs liés aux JV, notamment ceux qui présentent du contenu violent, ont également été observés dans des études (Brun, 2017). En effet, ces derniers auraient tendance à diminuer l'empathie chez les utilisateurs (Bailey \& West, 2013) et, à l'inverse, lesjeuxconsidérés comme "prosociaux» favoriseraient la tendance aux comportements charitables (Saleem, Anderson, \& Gentile, 2012). Ainsi, si les jeux de tir à la première personne améliorent les capacités d'attention visuospatiale, en contrepartie, ils diminuent les capacités de discernement d'émotions faciales dites positives (Bailey \& West, 2013).

En bref, I'utilisation des JV dans un contexte thérapeutique nécessite une vigilance pour minimiser les effets nuisibles tels que ceux retrouvés dans les $\mathrm{JV}$ trop violents. Aussi, si certains JV présentent un intérêt pour les sujets neurotypiques en stimulant certaines fonctions, d'autres semblent avoir des effets délétères. Par conséquent, l'enjeu mérite qu'on s'y penche pour connaitre les effets des JV chez des populations présentant certaines pathologies et, plus particulièrement, chez des populations ayant un TSA (Brun, 2017). Comme il sera abordé dans le présent texte, certains chercheurs dans ce domaine suggèrent que l'utilisation de ces technologies chez les individus avec un TSA peut favoriser la communication et I'inclusion sociale (Pottelette, 2016), tandis que d'autres, au contraire, considèrent qu'il y aurait un plus grand risque d'utilisation excessive des outils informatiques ainsi que de l'isolation sociale (Grynszpan, Weiss, \& Perz-Diaz, 2014).

\section{Les conséquences neurologiques chez les sujets neurotypiques}

Plusieurs équipes de chercheurs se sont penchées sur I'impact des JV sur le fonctionnement cérébral. Pour ce faire, des études ont utilisé l'imagerie ou l'électroencéphalogramme afin d'observer les modifications de l'activité cérébrale chez des joueurs comparativement à celles des non-joueurs. Ces études démontrent que, chez les utilisateurs de JV, il y a une diminution de l'activation au niveau du cortex préfrontal dorsolatéral droit après l'entraînement (Brun, 2017). Conséquemment, selon Lee et coll. (2012), ces changements d'activation sont liés à de meilleures capacités d'orientation visuospatiale. Kühn, Gleich et coll. (2013) ont constaté, d'un point de vue morphologique plutôt que fonctionnel, une augmentation du volume du cortex préfrontal dorsolatéral droit, de la section droite de l'hippocampe et du cervelet, et ce, lors de la pratique d'un jeu de plateforme commercial tel que 
le jeu Super Mario 640. Ces régions cérébrales sont associées à la navigation spatiale, à la planification des actions, à la mémoire de travail ainsi qu'aux performances motrices (Kühn et coll., 2013).

Lors du jeu, il y aurait aussi une modification de l'activation des zones cérébrales associées à la mémoire de travail, à l'attention et au contrôle moteur durant leurs sessions de JV. Ces altérations d'activation cérébrale seraient associées à des réponses plus rapides des centres de la vision, notamment dans le cortex occipital, responsable du traitement des informations visuelles (Latham, Patston, Westermann, Kirk, \& Tippett, 2013). Également, ces modifications de l'activation cérébrale mesurées lors de la pratique d'un jeu seraient également retrouvées chez le joueur au repos. Autrement dit, les zones activées lors du jeu sont parfois réactivées chez le joueur au repos. L'activation de certaines zones cérébrales lors du jeu accompagnée parfois d'une réactivation chez le joueur au repos améliore d'autant plus ses capacités, dont celles concernant les réponses plus rapides des centres de la vision (Martinez, Solana, Burgaleta, Hernández-Tamames, Alvarez-Linera, Román et coll., 2013). De plus, la pratique des JV influe aussi sur les circuits de la récompense, puisque certaines études notent une activation du striatum, et ce, que ce soit pendant ou après leur utilisation (Lorenz, Gleich, Gallinat, \& Kühn, 2015).

Ensuite, un volume supérieur du cortex pariétal postérieur droit chez les joueurs serait associé à de meilleures capacités visuospatiales (Tanaka, Ikeda, Kasahara, Kato, Tsubomi, Sugawara et coll., 2013). Également, le même phénomène est retrouvé au niveau des gyrus frontaux supérieur, pariétal supérieur et précentral droits qui sont associés à de meilleures capacités de flexibilité mentale, de raisonnement abstrait et de formation de concepts et stratégies de réponse à l'évolution des contingences contextuelles. Il en est de même pour le volume des cortex préfrontal dorsolatéral et frontal gauche qui augmenterait, ceux-ci étant associés au centre des contrôles exécutifs, de la planification des actions et de la gestion du champ visuel. Le phénomène est encore une fois retrouvé dans l'insula gauche, associée à l'attention et aux fonctions sensori-motrices (Tanaka et coll., 2013).

En bref, ces études attestent des modifications du fonctionnement et du volume cérébral en rapport avec la pratique des JV. Néanmoins, les changements notés au niveau de certaines régions varient selon les études. En effet, il y a des différences quant aux localisations anatomiques évaluées, ainsi qu'au sens (diminution ou augmentation de l'activité cérébrale) de la modification retrouvée. Afin de réconcilier ces incongruences liées au sens du changement de l'activité cérébrale, certains auteurs considèrent qu'une diminution d'activation est liée à une augmentation des capacités sous-tendues par ces régions cérébrales (Lee, Voss, Prakash, Boot, Vo, Basak et coll., 2012). Cette logique s'explique par le fait que le «cerveau aurait moins besoin de se mobiliser pour effectuer une même action et donc serait plus efficient» (Brun, 2017). Au contraire, certaines études observent plutôt une augmentation d'activation corrélée avec une augmentation de la fonction associée (Kühn \& coll., 2014; Tanaka \& coll., 2013). En somme, la pratique des JV a vraisemblablement un impact sur le cerveau des joueurs neurotypiques. Les limites de cette influence ne sont toutefois pas complètement établies en raison des connaissances incomplètes sur le fonctionnement du cerveau (Brun, 2017).

\section{Les particularités cérébrales des sujets avec un TSA}

Le cerveau des individus avec un TSA a d'abord été considéré comme identique à celui des individus neurotypiques. En réalité, le cerveau des sujets avec un TSA présente des anomalies concernant la trajectoire neuro-développementale cérébrale et la connectivité cérébrale (Pottelette, 2016). À cela s'ajoutent des anomalies fonctionnelles, c'est-à-dire une modification d'activation de certaines régions cérébrales, qu'elle soit plus élevée ou moindre que la normale. Autrement dit, les anomalies structurales entraînent parfois, chez les individus avec un TSA, une activation différente soit plus grande ou moindre de la région cérébrale ayant l'anomalie, et ce, dans des situations particulières.

Selon la revue de la revue de la littérature d'Ecker, Bookheimer et Murphy (2015), les anomalies concernant la trajectoire neuro-développementale cérébrale chez les sujets avec un TSA varient en fonction des régions cérébrales. Celles qui sont impliquées dans les symptômes diagnostiques du TSA seraient: les régions fronto-temporales et fronto-pariétales, le complexe amygdale hippocampe, le cervelet, les ganglions 
de la base et les régions cingulaires antérieures et postérieures. D'ailleurs, certaines anomalies de régions cérébrales spécifiques pourraient être associées à des symptômes cliniques spécifiques aux sujets avec un TSA. Les aires de Broca et de Wernicke, par exemple, ont été associées avec des déficits de la communication sociale et du langage. Les régions fronto-temporales et l'amygdale ont été, quant à elles, associées aux anomalies de la régulation socioémotionnelle. Finalement, le cortex orbitofrontal et le noyau caudé ont été associés aux comportements répétitifs et stéréotypés (Ecker et coll., 2015).

En ce qui concerne l'activation cérébrale, des anomalies structurales dans certaines régions ont été trouvées. Entre autres, des diminutions de l'activation des régions qui regroupent le réseau du cerveau social ont été notées lors de tâches concernant le processus émotionnel ou la cognition sociale (Ecker et coll., 2015). Plus précisément, ces diminutions d'activation des régions qui concerne le processus émotionnel ou la cognition touchent l'amygdale, la jonction temporo-pariétale, l'insula et le cortex frontal inférieur (Ecker et coll., 2015). Cependant, des augmentations anormales de l'activation cérébrale ont été notées en réponse à des stimuli négatifs et au regard direct (Ecker et coll., 2015). De plus, selon la revue de littérature de Boddaert et Zilbovicius (2002), des études d'activation ont observé un motif anormal de l'activation corticale chez les sujets avec un TSA. Ces résultats évoquent qu'il y aurait peut-être, chez les individus avec un TSA, différentes connexions entre certaines régions corticales (Boddaert \& Zilbovicius, 2002). Enfin, Sebastian et Blakemore (2011), à la suite d'études sur la réponse cérébrale au rejet social chez les adolescents avec un TSA, ont suggéré que «les circuits neuronaux impliqués dans la réponse au rejet social chez les sujets neurotypiques (par rapport à l'inclusion) étaient hypoactifs chez les adolescents avec un TSA» (Sebastian \& Blakemore, 2011, cités dans Pottelette, 2016).

En somme, le cerveau des individus avec un TSA présente d'abord des anomalies concernant la trajectoire neurodéveloppementale cérébrale et la connectivité cérébrale. Celles-ci varient en fonction des régions cérébrales. Autrement dit, certaines anomalies des régions cérébrales sont associées aux symptômes diagnostiques du TSA, par exemple, les régions frontotemporales et l'amygdale ont été associées aux anomalies de la régulation socioémotionnelle. Le cerveau des individus avec un TSA présente aussi des anomalies fonctionnelles. Ces dernières montrent une augmentation ou une diminution d'activation de certaines régions cérébrales. Ces régions cérébrales, étant liées à des fonctions, sont alors associées, tel a été le cas avec les anomalies de trajectoire neurodéveloppementale et de connectivité cérébrale, aux symptômes diagnostiques du TSA.

\section{L'impact des jeux vidéos chez les sujets avec un TSA}

De manière générale, les études concernant les impacts des JV sur les sujets avec un TSA utilisent des enfants, des adolescents ou des adultes comme échantillon. Il est rare qu'une étude traite de ces trois stades du développement et des conséquences d'un même type de JV afin de voir si les mêmes conséquences sont retrouvées indépendamment du stade de développement. Pottelette (2016) consacre une partie de sa thèse à une revue de la littérature sur le TSA et la réalité virtuelle. Cependant, il est souvent question des sujets avec TSA sans mention directe du stade de développement desdits sujets. Ainsi, dans la présente section, plusieurs études sont présentées et elles montrent différents impacts des JV sur les sujets avec un TSA. II est important de ne pas généraliser les résultats d'études des adultes sur les enfants ou les adolescents et l'inverse est tout aussi vrai.

Si la population générale utilise davantage Internet, il existe peu de données disponibles quant à l'utilisation d'Internet des individus avec un TSA qu'ils soient adultes, adolescents ou enfants. De même, peu d'études se penchent sur l'utilisation des JV chez la clientèle avec un TSA (Sallafranque St-Louis, 2015). Toutefois, il semble que les individus avec un TSA utilisent Internet comme un moyen de socialisation et qu'une de leurs activités de prédilection soit les jeux en ligne (Sallafranque St-Louis, 2015). Selon la revue de littérature de Georgescu, Kuzmanovic, Roth, Bente et Vogeley (2014), le fait d'utiliser des personnages virtuels, appelés des avatars, est un outil efficace pour les individus avec un TSA afin d'améliorer des compétences sociales ainsi que le décodage non verbal puisqu'ils procurent un environnement d'apprentissage sécurisant, reproductible et diversifiable. En ce sens, selon la revue de littérature de Parsons et Cobb (2011), 
I'utilisation de la réalité virtuelle chez les individus avec un TSA offre de nombreux avantages, par exemple, elle facilite le contrôle des stimuli et la compréhension, améliore les motivations de l'utilisateur à accomplir les tâches dans les paramètres de jeu, permet de personnaliser l'interface selon les besoins de son utilisateur et améliore les compétences sociales par rétrocontrôle en temps réel. Ainsi, les environnements virtuels ont plusieurs effets sur les personnes ayant un TSA, notamment, ils leur permettent une «maîtrise du jeu plus facile que les situations rencontrées dans la vie réelle» (Parsons \& Cobb, 2011, cités dans Pottelette, 2016, p. 35), la possibilité d'une communication qui n'est pas en face à face - ce que plusieurs sujets avec un TSA trouvent menaçant - puisque les utilisateurs peuvent communiquer par l'entremise de leurs avatars, et le jeu favoriserait les capacités de généralisation de l'acquis (Parsons \& Cobb, 2011).

De nombreux bénéfices sont donc associés plus précisément à la réalité virtuelle. En effet, on note des améliorations des mesures sociales et cognitives de la théorie de l'esprit et de la reconnaissance des émotions, et ce, que ce soit en milieu virtuel ou réel après I'utilisation des JV (Pottelette, 2016). De plus, l'exergaming, un type de jeux de réalité virtuelle qui combine l'exercice physique et mental, favorise une diminution des comportements répétitifs et une amélioration des fonctions cognitives (Pottelette, 2016). En bref, les jeux en ligne ainsi que les jeux de réalité virtuelle améliorent tous deux les compétences sociales des individus avec un TSA par leur environnement d'apprentissage sécurisant. D'une part, les jeuxen ligne améliorent le décodage non verbal et, d'autre part, la réalité virtuelle permet une meilleure généralisation des acquis, notamment par une amélioration de la reconnaissance des émotions en contexte réel ou virtuel ainsi qu'une amélioration des mesures sociales et cognitives de la théorie de l'esprit.

Cependant, la relation entre les individus avec un TSA et la réalité virtuelle possède des limites, notamment des risques d'addiction et d'isolement social. La méta-analyse de Grynszpan, Weiss et Perz-Diaz (2014) mentionne d'ailleurs que plusieurs auteurs ont noté les risques de dépendance et $d$ 'isolement social des enfants avec un TSA associés aux activités sur I'ordinateur. Selon la revue de littérature de GarciaZapirain et coll. (2014), les outils de réalité virtuelle peuvent entraîner de la dépendance et conduire davantage à de l'isolement. Dans un même ordre d'idées, certains programmes de réalité virtuelle "[peuvent] isoler socialement un enfant dans les cas où il n'interagissait qu'avec une machine et non avec une autre personne» (Ploog et coll., 2013, cités dans Pottelette, 2016, p. 41).

Plus spécifiquement, le risque d'addiction aux JV chez les sujets avec un TSA a été examiné par quelques études de cas-témoin. Celle de Mazurek et Engelhardt (2013) a cherché à comparer l'utilisation problématique des JV chez des enfants et des adolescents avec un TSA et chez des enfants et des adolescents groupe témoin. Les résultats ont démontré une utilisation plus problématique des $\mathrm{JV}$ chez les enfants et les adolescents avec un TSA comparativement à ceux témoins. Ces résultats font un parallèle avec ceux trouvés dans l'étude cas-témoin de Macmullin, Lunsky et Weiss (2015), qui analysait les profils et les conséquences de l'utilisation des outils électroniques chez des sujets entre 6 et 21 ans avec un TSA comparativement à des sujets témoins. Les résultats démontrent, entre autres, que les enfants et adolescents avec un TSA semblaient avoir une utilisation plus compulsive des JV par rapport aux sujets témoins. Par ailleurs, les résultats des deux échantillons démontrent que les garçons utilisaient davantage les JV que les filles. De plus, des questionnaires concernant les conséquences de l'utilisation des outils électroniques chez les enfants et adolescents entre 6 et 21 ans ont été remplis par des parents ayant des enfants et adolescents avec un TSA et par des parents ayant des enfants neurotypiques. Les résultats démontrent que les parents des enfants du groupe avec un TSA ont «significativement déclaré plus fréquemment que l'utilisation d'outils électroniques avait un impact négatif sur leurs enfants» (Macmullin et coll., 2015, cités dans Potelette, 2016, p. 41).

Également, comme il a été vu précédemment, les JV violents auraient tendance à mener à une diminution des capacités d'empathie chez leurs utilisateurs, c'est-à-dire par une diminution des capacités de discernement d'émotions faciales positives. Un questionnement subsiste quant aux conséquences neuropsychologiques que peuvent avoir ces types de jeux chez les individus avec un TSA, c'est-à-dire qu'on peut se demander si les JV violents pourraient diminuer davantage l'empathie des sujets avec un TSA. À l'opposé, est-ce que les jeux prosociaux entraîneraient 
le même type de bénéfice pour les individus avec un TSA? Pareillement, il est à se demander si les conséquences neurologiques subies par les sujets neurotypiques à la suite de I'utilisation des JV sont similaires chez les sujets avec un TSA. Si tel est le cas, est-ce que les changements d'activation entraîneraient les mêmes changements sur le plan du fonctionnement? En effet, si le cerveau des individus avec un TSA possède déjà des particularités, ces changements auront-ils le même effet chez des sujets avec un TSA que chez des sujets neurotypiques? En fait, l'impact des JV chez les individus avec un TSA est une branche de la recherche qui est encore en évolution, notamment en ce qui concerne leurs conséquences neurologiques et neuropsychologiques dans un contexte de divertissement ainsi que dans un contexte thérapeutique.

Sachant que les JV sont utilisés par une majorité d'Européens et qu'ils sont utilisés à la fois par des individus neurotypiques et des individus avec un TSA, il est primordial d'évaluer les impacts qu'ils peuvent entraîner afin de mieux comprendre les effets neurologiques et neuropsychologiques qu'ils peuvent avoir chez les sujets neurotypiques et chez les sujets avec un TSA. Ensuite, il sera possible de faire des prédictions plus précises quant aux effets possibles lorsqu'utilisés en tant que moyens thérapeutiques chez les individus avec un TSA. II n'empêche que, même s'il existe peu d'études qui étudient l'effet des JV employés en contexte $d$ 'intervention pour la reconnaissance des émotions ou du développement de diverses habiletés cognitives ou encore socioémotionnelles, il est fort improbable que les JV utilisés en contexte thérapeutique soient à risque de développer une dépendance chez ses utilisateurs. Ils ne renferment pas les caractéristiques de jeux "addictifs» conçus pour le divertissement (p.ex., avec jeux de rôles et avatars, multijoueurs, pénalité si on décroche avant la fin d'une partie). De plus, leur utilisation ne se fait pas pour les motifs associés à la cyberdépendance (p.ex., jouer pour éviter, jouer pour fuir la réalité ou jouer par compétitivité). En outre, ils sont monitorés de plus près par les parents et les intervenants, ce qui permet d'en décrocher plus facilement.

\section{Conclusion}

Somme toute, le diagnostic du TSA retrouvé dans la cinquième édition du DSM renvoie à une notion de spectre possédant deux sphères de symptômes: l'altération sociocommunicationnelle ainsi que le caractère répétitif et stéréotypé des comportements et des intérêts. II existe des programmes thérapeutiques pour les individus avec un TSA, dont certains utilisent les technologies numériques. Si certaines études évaluent les conséquences de ces traitements sur les individus avec un TSA, peu tentent d'étudier les conséquences des technologies numériques, dont les JV, dans un contexte non thérapeutique. En effet, il est important de comprendre les effets neuropsychologiques et neurologiques de JV chez les individus avec un TSA dans un contexte de divertissement pour ainsi mieux prédire les effets de I'utilisation de JV comme modalité thérapeutique.

Des études ont observé, chez les individus neurotypiques, des conséquences neuropsychologiques et neurologiques en lien avec la pratique de JV. En ce sens, les conséquences neuropsychologiques de certains types de $\mathrm{JV}$ touchent les fonctions cognitives associées à la vision comme la mémoire visuelle de travail (Brun, 2017). Toutefois, d'autres types de $\mathrm{JV}$ influenceraient les capacités d'empathie et de discernement d'émotions faciales positives (Bailey \& West, 2013). Les conséquences neurologiques des JV sur les sujets neurotypiques sont composées de modifications du fonctionnement et du volume cérébral (Ecker, Bookheimer, \& Murphy, 2015). Du côté des individus avec un TSA, la pratique de JV serait plus en mesure, entre autres, d'améliorer leurs compétences sociales, le décodage non verbal, le contrôle des stimuli ainsi que la compréhension par l'utilisation de la réalité virtuelle (Parsons \& Cobb, 2011). Cependant, des risques d'addiction et d'isolement découlant de I'utilisation des JV sont présents (Grynszpan et coll., 2014). D'ailleurs, des études cas-témoin observent un risque d'utilisation compulsive des JV plus élevé chez les sujets avec un TSA comparativement aux sujets neurotypiques (Mazurek \& Engelhardt, 2013). Ainsi, il semble exister, tout comme chez des individus neurotypiques, des risques de dépendance aux JV. $D$ 'autres recherches sont néanmoins nécessaires afin d'approfondir nos connaissances sur les conséquences neurologiques et neuropsychologiques de la pratique des JV chez les individus avec un TSA afin de pouvoir prédire les effets de ceux-ci dans un contexte thérapeutique. 


\section{Références}

American Psychiatric Association. (2013). DSM-V: Manuel diagnostique et statistique des troubles mentaux $\left(5^{\mathrm{e}}\right.$ éd.). Issy-les-Moulineaux, France: Elsevier Masson.

Bailey, K., West, R. (2013). The effects of an action video game on visual and affective information processing. Brain Research. 1504, 35-46. https://doi. org/10.1016/j.brainres.2013.02.019

Beaulne, S. (2012). La conceptualisation de l'autisme depuis Kanner: où en sommes-nous? Le Journal Sur Les Handicaps Du Développement, 18(1), 43-61. Récupéré de http://oadd.org/wp-content/ uploads/2012/01/41011_JoDD_18-1_43-61_Beaulne.pdf

Boddaert, N., \& Zilbovicius, M. (2002). Functional neuroimaging and childhood autism. Pediatric Radiology, 32(1), 1-7. https://doi.org/10.1007/s00247-001-0570-x

Bolduc, M. \& Poirier, N. (2017). La démarche et les outils d'évaluation clinique du trouble du spectre de l'autisme à l'ère du DSM-5. Revue de psychoéducation. 46(1), 73-97. https://doi.org/10.7202/1039682ar

Brun, G. (2017). Utilisation des jeux vidéo dans les soins de réhabilitation des sujets souffrant de schizophrénie: revue de la littérature (Thèse de doctorat, Université de Bordeaux, Bordeaux, France). Récupéré de https://pdfs.semanticscholar.org/ dbe7/f3902dab9584ce1b8c3c6163284586020b58.pdf

Ecker, C., Bookheimer, S. Y., \& Murphy, D. G. (2015). Neuroimaging in autism spectrum disorder: brain structure and function across the lifespan. The Lancet Neurology, 14(11), 1121-1134. https://doi. org/10.1016/S1474-4422(15)00050-2

Eckert, A. (2019). Accompagnement des enfants et des adolescents en âge scolaire présentant des TSA. Sécurité social CHSS, 2, 28-32. Récupéré de https://soziale-sicherheit-chss.ch/wp-content/ uploads/2019/06/fr_BSV_CHSS_02-19_Eckert.pdf

Georgescu, A. L., Kuzmanovic, B., Roth, D, Bente, G., \& Vogeley, K. (2014). The use of virtual characters to assess and train non-verbal communication in high-functioning autism. Frontiers in Human Neuroscience. 8, 807. https://doi.org/10.3389/ fnhum.2014.00807

Glass, B. D., Maddox, W. T., Love, B. C. (2013). Real-time strategy game training: emergence of a cognitive flexibility trait. PloS One. 8(8): e70350. https://doi. org/10.1371/journal.pone.0070350
Grossard, C., \& Grynszpan, O. (2015). Entraînement des compétences assistées par les technologies numériques dans l'autisme: une revue. Enfance. 1, 67-85. https://doi.org/10.4074/S0013754515001056

Grynszpan, O.,Weiss, P.L., \&Perz-Diaz, F. (2014). Innovative technology-based interventions for autism spectrum disorders: A meta-analysis. Autism, 18(4), 346-361. https://doi.org/10.1177/1362361313476767

Kühn, S., Gleich, T., Lorenz R. C., Lindenberger, U., \& Gallinat, J. (2014). Playing Super Mario induces structural brain plasticity: gray matter changes resulting from training with a commercial video game. Molecular Psychiatry, 19, 265-271. https:// doi.org/10.1038/mp.2013.120

Kuss, D. J. et Griffiths, M. D. (2012). La dépendance aux jeux vidéo sur internet: une revue systématique des recherches empiriques disponibles dans la littérature. Adolescence, 1(79), 17-49. https://doi. org/10.3917/ado.079.0017

Latham, A. J., Patston, L. L. M., Westermann, C., Kirk, I. J., Tippett, L. J. (2013). Earlier Visual N1 Latencies in Expert Video-Game Players: A Temporal Basis of Enhanced Visuospatial performance? PloS One, 8(9): e75231. https://doi.org/10.1371/journal.pone.0075231

Lee, H., Voss, M.W., Prakash, R.S., Boot, W.R., Vo, L.T.K., Basak, C., ... Kramer, A. F. (2012). Videogame training strategy-induced change in brain function during a complex visuomotor task. Behavioural Brain Research, 232(2), 348 357. https://doi. org/10.1016/j.bbr.2012.03.043

Lorenz, R. C., Gleich, T., Gallinat, J., Kühn, S. (2015). Video game training and the reward system. Frontiers in Human Neuroscience, 9, 40. https://doi. org/10.3389/fnhum.2015.00040

Macmullin, J. A., Lunsky, Y., \& Weiss, J. A. (2015). Plugged in: Electronics use in youth and young adults with autism spectrum disorder. Autism, 20(1), 45-54 https://doi.org/10.1177/1362361314566047

Martínez, K., Solana, A. B., Burgaleta, M., HernándezTamames, J. A., Alvarez-Linera, J., Román, F. J., et al. (2013). Changes in resting-state functionally connected parietofrontal networks after videogame practice. Human Brain Mapping, 34(12), 3143-3157. https://doi.org/10.1002/hbm.22129

Mazurek, M. O., \& Engelhardt, C. R. (2013). Video Game Use in Boys With Autism Spectrum Disorder, ADHD, or Typical Development. Pediatrics, 132(2), 260-266. https://doi.org/10.1542/peds.2012-3956 
Morasse, K. (2015). Spectre autistique: Autisme, Asperger, TED, TSA: comment s'y retrouver?. Association québécoise des neuropsychologues. Récupéré de https://aqnp.ca/documentation/developpemental/ le-spectre-autistique/

Organisation mondiale de la Santé (2018). Trouble du jeu vidéo. OMS. Récupéré de https://www.who.int/features/qa/gaming-disorder/fr/

Parsons, S., \& Cobb, S. (2011). State-of-the-art of Virtual Reality technologies for children on the autism spectrum. European Journal of Special needs Education, 26(3), 355-366. https://doi.org/10.1080/08856257.2 011.593831

Ploog, B. O., Sscharf, A., \& Nnelson, D. (2013). Use of Computer-Assisted Technologies (CAT) to Enhance Social, Communicative, and Language Development in Children with Autism Spectrum Disorders. Journal of Autism and Developmental Disorders, 43(2), 301-322. https://doi.org/10.1007/s10803$012-1571-3$

Pottelette, J. (2016). Troubles du spectre autistique et réalité virtuelle. Risque de pratique excessive des jeux vidéo chez les adolescents avec TSA (Thèse de doctorat, Université Paris Descartes, Paris, France). Récupéré de https://core.ac.uk/download/ pdf/52191398.pdf

Rufat S., Minassian H., \& Coavoux S. (2014). Jouer aux jeux vidéo en France. L'Espace Géographique, 43(4), 308-323. https://doi.org/10.3917/eg.434.0308.

Saleem, M., Anderson, C. A., Gentile, D. A. (2012). Effects of Prosocial, Neutral, and Violent Video Games on Children's Helpful and Hurtful Behaviors. Aggressive Behavior. 38(4), 281-287.
Sallafranque St-Louis, F. (2015). L'utilisation d'Internet et la solicitation sexuelle sur le Web auprès des personnes avec une déficience intellectuelle (DI) ou un trouble du spectre de l'autisme (TSA). Université du Québec en Outaouais. Récupéré de http://di.uqo.ca/818/1/Essai_Francois_Sallafranque St-Louis_2015.pdf

Sebastian, C. L., \& Blakemore, S.-J. (2011). Understanding the neural response to social rejection in adolescents with autism spectrum disorder: A commentary on Masten et al., McPartland et al., and Bolling et al. Developmental Cognitive Neuroscience, 1(3), 256259. https://doi.org/10.1016/j.dcn.2011.03.006

Schumann, C. M., Bloss, C. S., Barnes, C. C., Wideman, G. M., Caper, R. A., Akshoomoff, N., ... Courchesne, E. (2010). Longitudinal Magnetic Resonance Imaging Study of Cortical Development through Early Childhood in Autism. Journal of Neuroscience, 30(12), 4419-4427. https://doi.org/10.1523/JNEUROSCI.5714-09.2010

Tanaka, S., Ikeda, H., Kasahara, K., Kato, R., Tsubomi, H., Sugawara, S. K., ... Watanabe, K. (2013). Larger right posterior parietal volume in action video game experts: a behavioral and voxel-based morphometry (VBM) study. PLoS ONE. 8(6), e66998. https:// doi.org/10.1371/journal.pone.0066998

Zablotsky, B., Black, L., Maener, M., Schieve, L., \& Blumberg, S. (2015). Estimated Prevalence of Autism and Other Developmental Disabilities Following Questionnaire Changes in the 2014 National Health Interview Survey. National Health Statistics Reports. Récupéré de https://stacks.cdc.gov/view/cdc/38790

\section{Pour citer l'article}

Couture, K. (2020). Pratique excessive des jeux vidéo chez les personnes ayant un trouble du spectre de l'autisme. Psycause: Revue scientifique étudiante de l'École de psychologie de l'Université Laval, 10(1), 18-27.

\section{Droits d'auteur}

(C) 2020 Couture. Cet article est distribué en libre accès selon les termes d'une licence Creative Commons Attribution 4.0 International (de type CC-BY 4.0) qui permet l'utilisation du contenu des articles publiés de façon libre, tant que chaque auteur ou autrice du document original à la publication de l'article soit cité(e) et référencé(e) de façon appropriée. 